Pour paraphraser Karl Marx,

« un spectre hante la physique:

le spectre d'Ettore Majorana ».

Près de 80 ans après sa

disparition, les travaux du

physicien italien continuent

d'inspirer les chercheurs.

Ainsi, dans son dernier article,

Majorana prédit qu'une particule

élémentaire dépourvue de charge

électrique pourrait être sa propre

antiparticule [1], en opposition

avec la théorie de Dirac, pour

laquelle une particule et son

antiparticule sont distinctes.

Plusieurs groupes ont récemment annoncé la découverte de quasi-

particules de Majorana dans des

nanostructures supraconductrices.

\section{En plus d'être leur propre}

antiparticule, ces quasi-particules possèdent des propriétés

exotiques qui sont d'un grand

intérêt pour le développement

d'un ordinateur quantique

topologiquement protégé(a).

\title{
Le spectre de Majorana
}

Des quasi-particules exotiques découvertes dans des nanostructures supraconductrices pourraient servir
à construire un ordinateur quantique

Manuel Houzet ${ }^{(1)}$ (manuel.houzet@cea.fr), Julia Meyer ${ }^{(1)}$ (julia.meyer@univ-grenoble-alpes.fr) et Pascal Simon(2) (pascal.simon@u-psud.fr).

(1) Laboratoire Photonique, Électronique et Ingénierie Quantique (PHELIQS), Université Grenoble Alpes et CEA, 17 rue des Martyrs, 38054 Grenoble Cedex 9

(2) Laboratoire de Physique des Solides, CNRS, Univ. Paris-Sud, Univ. Paris-Saclay, Bât. 510, 91405 Orsay Cedex

\section{Des particules élémentaires aux quasi-particules} de la physique des solides

Les particules élémentaires décrites par la mécanique quantique sont classées en deux grandes familles : les fermions et les bosons. Les fermions, tels que les électrons ou les quarks dont sont constitués les protons et les neutrons, constituent la matière, tandis que les bosons, tels que les photons de l'électromagnétisme ou les gluons de l'interaction forte, sont les médiateurs des interactions. Chaque particule fermionique est associée à son antiparticule avec laquelle elle peut s'annihiler. Ainsi, lorsqu'un électron et un positron entrent en collision, ils peuvent disparaître en produisant deux photons. Selon le modèle standard qui est le cadre théorique pour comprendre la plupart des propriétés de la matière, les antiparticules sont distinctes des particules ; elles ont par exemple des charges électriques opposées. Ce n'est pas le cas pour les particules prédites par le physicien italien Majorana (fig. 1), depuis nommées « fermions de Majorana ", qui sont leur propre antiparticule. L'absence de charge électrique est donc une condition nécessaire pour envisager qu'une particule élémentaire possède cette propriété. Des expériences sont en cours, par exemple dans le laboratoire souterrain de Modane, pour identifier si les neutrinos qui sont les seuls fermions élémentaires dont la charge électrique est nulle sont des fermions ordinaires ou de Majorana [2]. Leurs résultats auront des conséquences importantes en physique des particules, pour comprendre par exemple

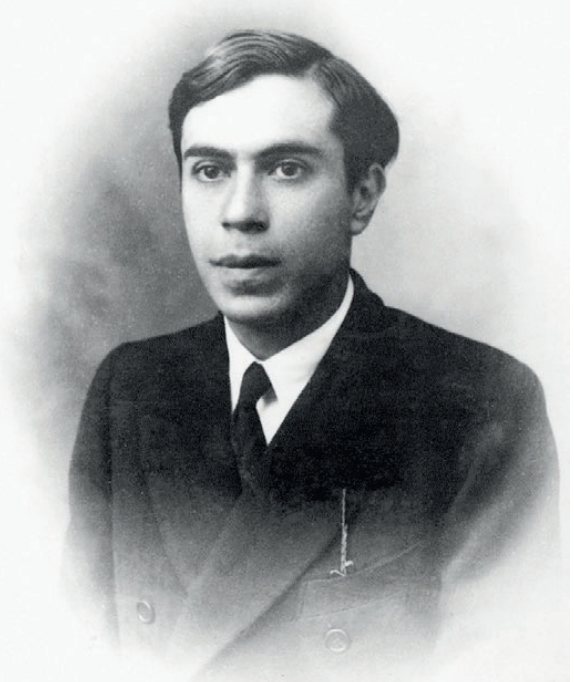

1. Photo d'Ettore Majorana. (Source : wikipedia) Né en 1906, Majorana est un physicien italien qui a apporté des contributions théoriques majeures dans les neuf articles qu'il a publiés. Sa disparition mystérieuse en 1938 a inspiré de nombreux écrivains [dont Friedrich Dürrenmatt, Les physiciens (1962), et Leonardo Sciascia, La disparition de Majorana (1975)].

l'origine de la faible masse des neutrinos, ou en cosmologie pour expliquer la dissymétrie matière/antimatière de notre Univers. Mais, à ce jour, la nature des neutrinos reste inconnue.

La physique des solides offre un terrain alternatif pour la découverte non pas de particules élémentaires, mais de " quasiparticules " aux propriétés exotiques. En effet, la plupart des propriétés de la matière proviennent des électrons, qui sont des fermions. Par contre, leur comportement 
dans un matériau n'est pas le même que celui des électrons élémentaires dans le vide. Dans un solide cristallin, chacun des atomes qui occupent les sites d'un réseau régulier libère des électrons qui peuvent s'y propager. Ces électrons itinérants interagissent entre eux, ainsi qu'avec les vibrations du réseau des atomes ionisés. Pour expliquer et prédire les propriétés d'un matériau, il est alors plus commode de décrire ces comportements collectifs comme étant ceux de "quasi-particules " indépendantes, et non comme la somme d'une multitude de comportements individuels, mais interdépendants, des électrons et des ions.
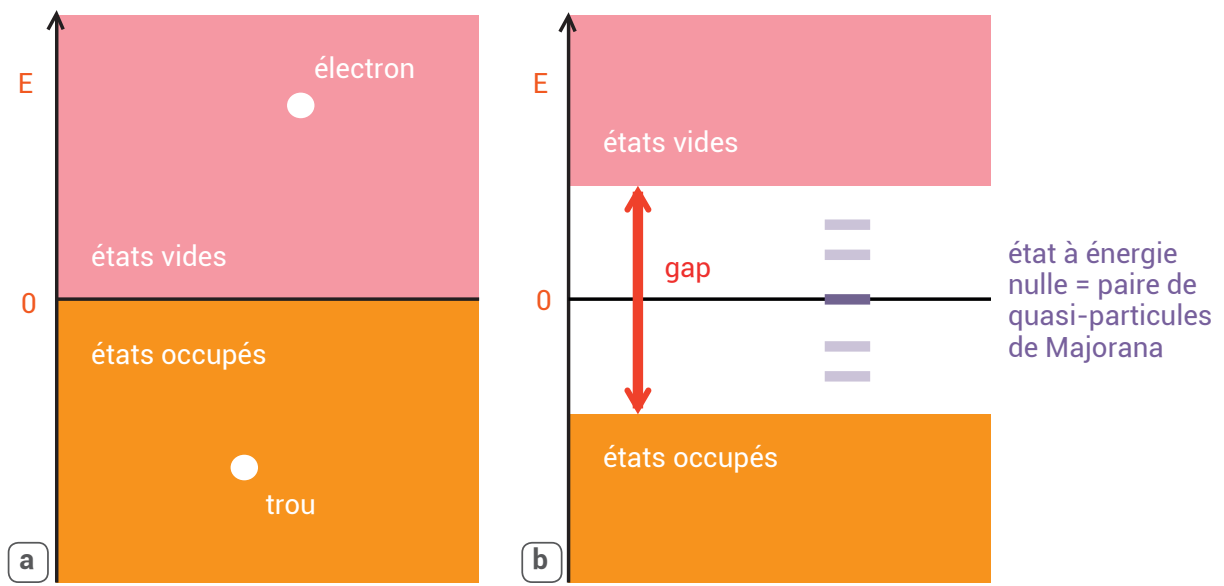

2. États (quasi) électroniques en fonction de l'énergie dans un métal et dans un supraconducteur. a) Dans un métal, les états d'énergie négative sont occupés - ils forment une mer de Fermi ; les états d'énergie positive sont vides. Les quasi-particules sont soit des électrons au-dessus de la mer de Fermi, soit des absences d'électrons (ou encore des trous) en dessous.

b) Dans un supraconducteur, les états mobiles occupés et vides sont séparés par une région d'énergies inaccessibles (ou gap) dans le spectre des excitations. Des paires d'états localisés peuvent apparaître dans le gap, tout en gardant des énergies opposées. Un supraconducteur topologique est caractérisé par l'apparition d'un unique état d'énergie nulle.
Souvent, les propriétés des quasi-particules restent similaires à celles des électrons dont es sont issues ; seuls certains paramètres inchangée. Ces électrons "habillés" forment alors un «liquide de Fermi », dont la théorie rend compte des propriétés de

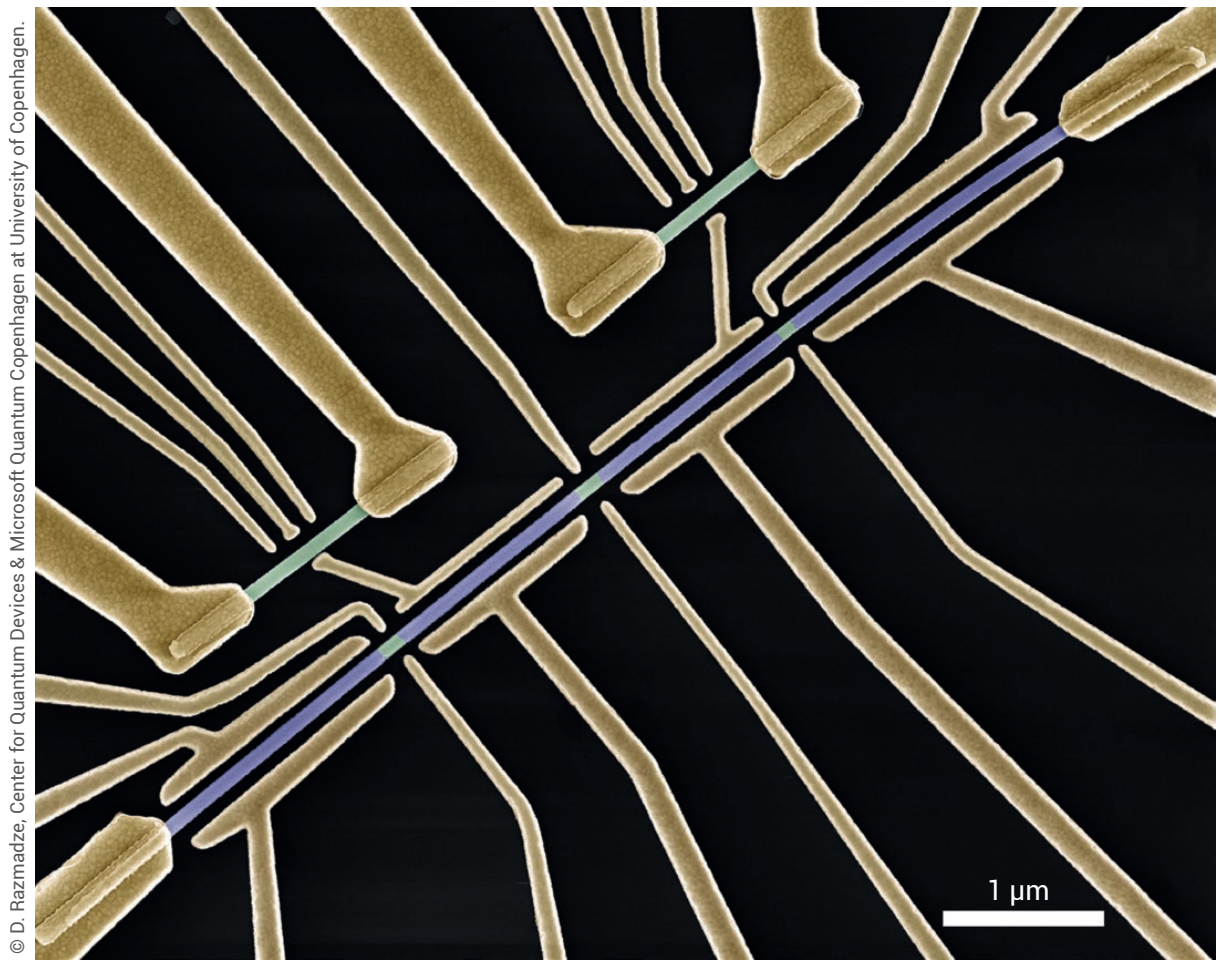

Image obtenue par microscopie électronique à balayage d'un circuit réalisé pour manipuler des quasi-particules de Majorana. Les couleurs ont été ajoutées pour indiquer le nanofil d'InAs (vert) recouvert d'aluminium (bleu) et contrôlé grâce à des grilles électrostatiques en titane et or (jaune).

nombreux métaux. Comme dans le gaz de Fermi étudié dans les cours universitaires de physique statistique, chaque quasiparticule fermionique occupe un " état quantique " avec une certaine énergie et une certaine impulsion; elle est donc délocalisée spatialement. De plus, deux fermions ne peuvent pas occuper le même état en raison du principe d'exclusion formulé par Pauli. D'après ces règles, l'état fondamental d'un système électronique, c'est-à-dire l'état de plus basse énergie, correspond à l'occupation de tous les états quantiques dont l'énergie est inférieure à une valeur spécifique, appelée niveau de Fermi. Par analogie avec un concept introduit par Dirac en physique des particules, on dit que ces états quantiques forment une "mer de Fermi ». Cette dernière peut être considérée comme un espace "vide ", caractérisé par l'absence de quasiparticules et dont l'énergie totale sert de référence pour la mesure des énergies. Les quasi-particules sont alors des états quantiques excités de la mer de Fermi. Un champ électrique appliqué a tendance à déplacer ces quasi-particules qui sont chargées. La résistance électrique des métaux a pour origine le principe de Pauli, qui freine la réorganisation de la mer de Fermi induite par ces déplacements. 
Les quasi-particules d'un liquide de Fermi se classent en deux catégories : des quasi-électrons d'énergie positive, qui occupent des états vides au-dessus du niveau de Fermi, et des quasi-électrons d'énergie négative, soustraits d'états occupés en dessous du niveau de Fermi (fig. 2a). Alternativement, on peut considérer ces lacunes de quasi-électrons comme la création de "quasi-trous " dans la mer de Fermi. Cette description des excitations d'un métal fonctionne aussi pour les semiconducteurs, qui sont caractérisés par une bande interdite - ou gap spectral - autour du niveau de Fermi. Comme un quasi-trou correspond à l'absence d'un quasi-électron, il a une charge opposée à celle de l'électron. En outre, un quasi-électron et un quasitrou peuvent s'annihiler : c'est un tel processus qui produit la lumière des diodes semi-conductrices électroluminescentes. De même que le positron est l'antiparticule de l'électron, le quasi-trou est l'antiparticule du quasi-électron.

Il arrive aussi que les quasi-particules pertinentes ne soient plus des fermions, mais des bosons. C'est le cas des magnons dans les isolants magnétiques. En revanche, il existe des situations où les quasiparticules ont des propriétés radicalement différentes de celles des électrons et des bosons. En présence d'un champ magnétique perpendiculaire à la couche, certains composés électroniques bidimensionnels présentent une phase caractérisée par une résistance électrique longitudinale (parallèle au champ électrique) nulle et une résistance transverse constante. L'effet Hall quantique entier, découvert par von Klitzing et qui lui a valu le prix de Nobel de physique en 1985, correspond à des plateaux de conductance $^{(\mathrm{b})}$ transverse qui sont des multiples entiers du quantum de conductance $e^{2} / h \approx 1 /(25813 \Omega)$, où $e$ est la charge élémentaire et $h$ la constante de Planck. Cet effet peut être expliqué dans le cadre de la théorie du liquide de Fermi. Ce n'est pas le cas de l'effet Hall quantique fractionnaire qui correspond à des plateaux aux fractions $p / q$ du quantum de conductance, dont la découverte et l'interprétation ont valu le prix de Nobel de physique à Störmer, Tsui et Laughlin en 1998. Laughlin a eu l'intuition que les quasiparticules de l'effet Hall quantique fractionnaire portent une charge électrique qui est une fraction de la charge de l'électron. Depuis, différentes expériences ont mis en évidence une telle charge fractionnaire.

\section{Quasi-particules de Majorana dans les supraconducteurs topologiques}

Un effort théorique et expérimental intense durant ces dernières années a permis de créer les conditions pour observer une nouvelle sorte de quasi-particules présentant certaines analogies, mais aussi des différences, avec les fermions de Majorana. En désaccord avec Albert Camus pour qui «mal nommer un objet, c'est ajouter au malheur de ce monde ", nous allons voir que ces quasi-particules possèdent des propriétés encore plus fascinantes que celles envisagées par Majorana.

Pour envisager la physique de Majorana, il faut trouver un système dans lequel les quasi-particules n'ont pas de charge électrique. Ceci peut être réalisé dans les supraconducteurs. La supraconductivité a été découverte en 1911 par Kammerlingh Onnes, qui a observé que la résistivité électrique de certains métaux devient nulle en dessous d'une température critique. Il a fallu près d'un demi-siècle pour que ce comportement soit compris grâce à la théorie développée par Bardeen, Cooper et Schrieffer, récompensés par le prix Nobel de physique en 1972. Ils ont en effet expliqué que l'interaction des électrons avec les phonons, qui sont les vibrations du cristal, génère une interaction attractive entre les électrons. Celle-ci peut devenir plus importante que l'interaction répulsive due à leurs charges de même signe. Dès lors, l'énergie de l'état fondamental d'un métal peut être abaissée par la création de paires d'électrons, aussi nommées paires de Cooper, qui gagnent ainsi une certaine énergie d'appariement.

Comme les paires de Cooper qui constituent l'état fondamental d'un supraconducteur sont des bosons, elles produisent des propriétés radicalement différentes de celles d'un métal. En particulier, elles forment un "condensat " libre de se déplacer sans résistance au sein du matériau. La création d'une quasi-particule au-dessus de ce nouvel état fondamental, par exemple en injectant un électron dans le supraconducteur, nécessite de fournir au minimum l'énergie d'appariement. De ce fait, comme dans les semi-conducteurs, un gap apparaît dans le spectre des excitations autour du niveau de Fermi (fig. 2b). Mais, contrairement aux semi-conducteurs, la formation du condensat de Cooper dans l'état fondamental s'accompagne d'une profonde modification de la nature des excitations : le processus d'appariement "superpose " les quasi-électrons et quasi-trous du métal au sens quantique (dans le même sens qui défie notre vision classique et permet au chat de Schrödinger d'être à la fois mort et vivant). Par conséquent, la charge des quasi-particules du supraconducteur n'est plus égale à la charge de l'électron (-e) ou du trou $(+e)$, mais peut prendre une valeur arbitraire entre les deux. De plus, ces quasiparticules délocalisées obéissent à une symétrie électron/trou : chaque état à énergie $\mathrm{E}$ est accompagné d'un état à énergie -E. Il n'y a donc plus de distinction claire entre particule et antiparticule, comme dans un métal ou un semi-conducteur.

On peut casser une paire de Cooper $d u$ condensat en créant deux quasi-particules, grâce, par exemple, à l'absorption d'un photon. Le processus inverse est également possible : deux quasi-particules d'un supraconducteur peuvent s'annihiler (en formant une paire de Cooper tout en émettant un photon), propriété qu'elles ont en commun avec les fermions de Majorana. Comme Monsieur Jourdain "(disant) de la prose sans (qu'il) en susse rien ", il y a donc longtemps que les spécialistes de la supraconductivité jouent avec des objets similaires à ceux imaginés par le physicien italien.

La situation est encore plus intéressante dans les supraconducteurs spatialement inhomogènes, en raison de leur taille finie ou de la présence de défauts. Dans de tels systèmes, en plus des états délocalisés dans tout l'échantillon et dont l'énergie excède le gap (représentés en orange et rose sur la figure $2 \mathrm{~b}$ ), des états localisés au voisinage des bords ou des défauts peuvent apparaitre dans la bande interdite. Tout comme les quasi-particules délocalisées des supraconducteurs, ces états localisés obéissent à la symétrie électron/trou : ils apparaissent à deux énergies opposées $E$ et $-E$, et sont représentés en violet sur la figure $2 b$. Cette symétrie autorise alors l'existence d'un unique état à $E=0$ (en violet foncé sur la figure 2b), qui correspond à une superposition quantique à poids égal d'électron et de trou. Ce sont de telles quasi-particules qui sont activement étudiées dans les nanostructures supraconductrices. Malheureusement, les états localisés d'énergie nulle n'existent pas de façon robuste dans les supraconducteurs standard, 

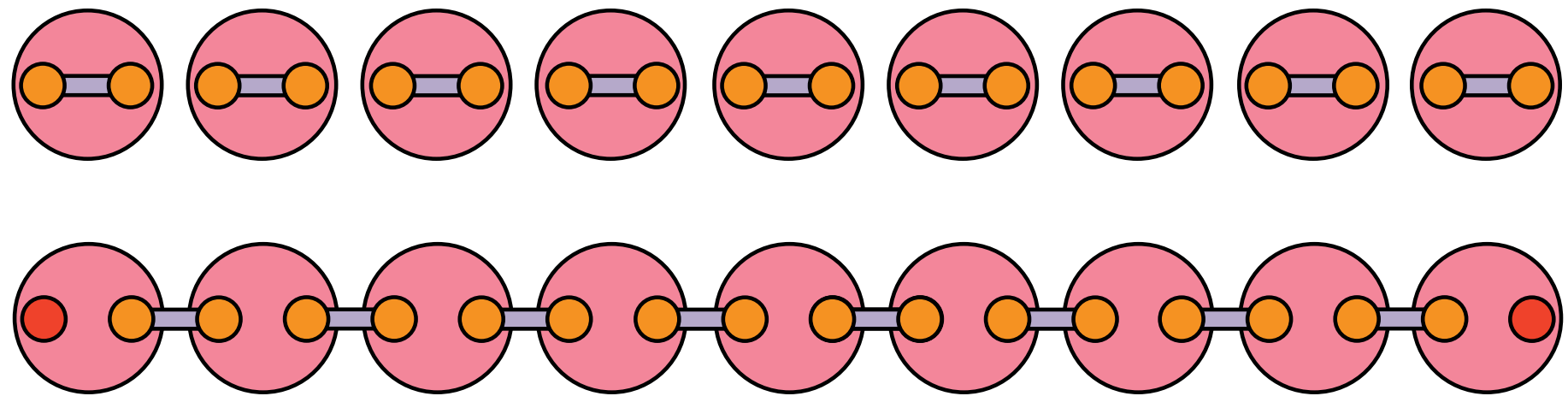

3. Modèle de Kitaev. Ce modèle explique la robustesse de l'état d'énergie nulle par sa nature fractionnaire. On peut imaginer chaque électron localisé aux sites d'une chaine comme la superposition de deux demi-électrons. Dans les supraconducteurs usuels (en haut), les deux demi-électrons d'un même site restent fortement liés. En revanche, dans un supraconducteur topologique (en bas), la liaison est réalisée entre deux demi-électrons de sites voisins. Il reste alors deux demi-électrons libres (ronds rouges) à chaque extrémité de la chaine : les quasi-particules de Majorana.

dont le spectre conserve un gap même en présence d'inhomogénéités. En revanche, on peut les trouver dans les supraconducteurs dits «topologiques".

La description la plus simple d'un matériau topologique est qu'il possède un gap autour du niveau de Fermi pour les états délocalisés dans tout son volume, ainsi que des états "topologiquement protégés " au niveau de Fermi et qui sont localisés à sa surface. Cette protection topologique des états de surface signifie qu'ils sont robustes à des perturbations de cette dernière et ne peuvent être détruits que si le gap se referme. De tels matériaux topologiques peuvent être soit des isolants (ou des semi-conducteurs) dont les états de surface sont métalliques, soit des supraconducteurs dont les états de surface sont associés avec des quasi-particules de Majorana. Peu après les travaux pionniers de Read et Green [3], un modèle simple de supraconducteur topologique qui permet de comprendre l'apparition d'un état de surface et son lien avec les quasi-particules de Majorana a été introduit par Kitaev [4] (fig. 3). Il s'agit d'une chaine de fermions sans degré de liberté interne qui peuvent se déplacer entre les sites d'un réseau unidimensionnel, en présence d'un potentiel attractif entre deux fermions localisés sur des sites adjacents. De façon générique, chaque fermion ordinaire peut être représenté par un nombre complexe, dont les parties réelle et imaginaire décrivent les deux quasi-particules de Majorana dont il est constitué : deux « demi-électrons ». Tant que ces deux quasi-particules de Majorana sont liées, une telle décomposition reste formelle, car elle n'a pas de conséquence mesurable. Kitaev a montré qu'il existe une phase de la chaine où deux quasi-particules de Majorana sur des sites voisins se recombinent pour former un état fermionique ordinaire avec une énergie finie. Il reste alors une quasi-particule de Majorana sans partenaire sur chacun des sites aux deux extrémités de la chaine. Ce sont ces deux quasi-particules de Majorana distantes l'une de l'autre qui forment l'état d'énergie nulle mentionné dans le paragraphe précédent et produisent le "spectre de Majorana » de la figure $2 \mathrm{~b}$.

La séparation spatiale des deux quasiparticules de Majorana qui forment l'état d'énergie nulle explique la protection topologique : l'énergie reste piégée au niveau de Fermi dans une chaine suffisamment longue, c'est-à-dire tant que les deux quasi-particules de Majorana sont suffisamment éloignées l'une de l'autre. Un état fermionique ordinaire d'énergie nulle est alors codé de façon non locale par deux quasi-particules de Majorana. On dispose ainsi d'un état fermionique "fractionné " en deux. Comme l'état d'énergie nulle peut être vide ou occupé, l'état fondamental d'un supraconducteur topologique est dégénéré. Il s'agit en fait d'une dégénérescence de parité du nombre d'électrons dans l'état fondamental d'un supraconducteur isolé électriquement. Dans un supraconducteur conventionnel, le condensat de paires de Cooper accueille un nombre pair d'électrons, tandis que l'ajout d'un électron coute une énergie au minimum égale au gap. La parité du nombre total d'électrons est donc fixée ${ }^{(c)}$. En revanche, dans un supraconducteur topologique, l'ajout d'un électron est réalisé en changeant l'occupation de l'état d'énergie nulle. La parité du nombre d'électrons dans l'état fondamental est donc arbitraire.

\section{Réalisations et signatures expérimentales}

La chaine de Kitaev n'est pas simple à réaliser avec un matériau supraconducteur. En effet, elle suppose que les fermions qui la constituent n'ont pas de degré de liberté interne. Or, les vrais électrons possèdent un spin, c'est-à-dire un moment magnétique intrinsèque qui peut pointer dans deux directions opposées. Pour lever cette dégénérescence de spin, on peut appliquer un champ magnétique. Malheureusement, celui-ci a également tendance à détruire la supraconductivité. D'autres voies doivent alors être explorées. Certains groupes se consacrent à l'étude de quelques rares matériaux supraconducteurs magnétiques qui pourraient être topologiques, mais qui ne sont faciles ni à synthétiser, ni à mesurer. La voie la plus prometteuse, qui est aussi majoritairement suivie, consiste à accoler un matériau supraconducteur à un autre qui ne l'est pas. En passant d'un matériau à l'autre, les électrons héritent des propriétés de chacun d'eux. Grâce à cet " effet de proximité ", on peut donc espérer induire une supraconductivité topologique dans un matériau non supraconducteur adapté [5]. En particulier, si le matériau possède un fort couplage spin-orbite - c'est-à-dire que les électrons s'y déplacent avec une vitesse qui dépend de la direction de leur spin, la levée de dégénérescence de spin pourra 


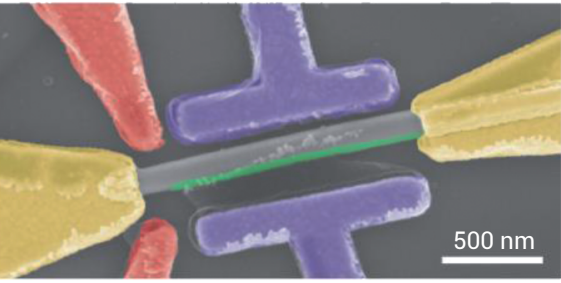

a

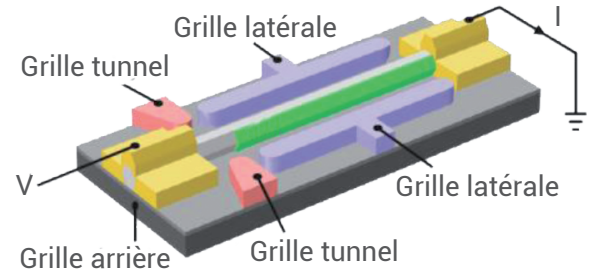

Grille arrière Grille tunne

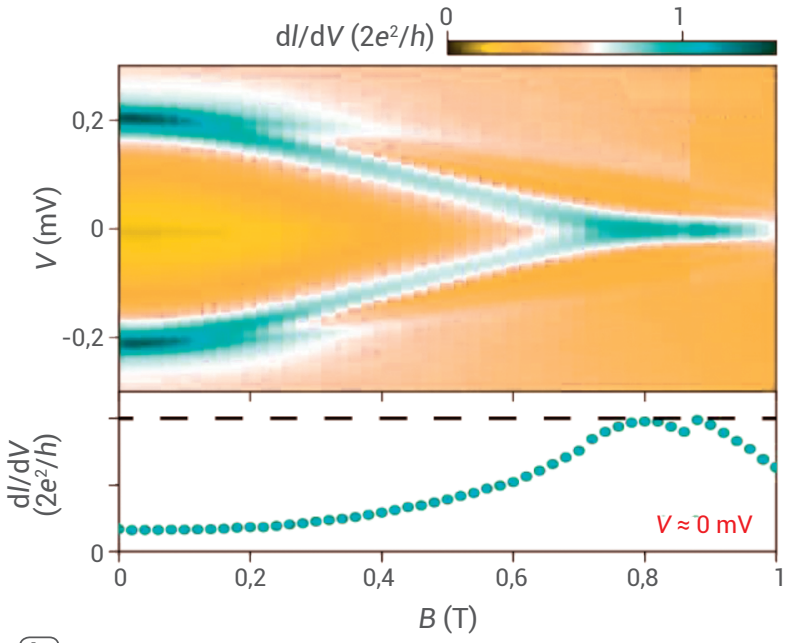

b

4. Quantification de la conductance d'un nanofil (source adaptée de la référence [14]).

a) Dispositif expérimental réalisé à Delft [14]. Un nanofil de InSb (gris) est enrobé par de l'aluminium (vert). Les deux extrémités sont connectées à des réservoirs métalliques (jaune) pour mesurer la conductance à travers le nanofil.

b) En haut : conductance $\mathrm{dl} / \mathrm{dV}$ en fonction du champ magnétique $B$ et de la tension de polarisation $V$, mesurée dans le dispositif montré en a). En bas : sur une plage de champ magnétique autour de $0,8 \mathrm{~T}$, on voit que la conductance à faible tension de polarisation atteint le quantum de conductance $2 e^{2} / h$. Cette conductance quantifiée est une signature attribuée à la présence d'une quasi-particule de Majorana localisée à l'extrémité du nanofil.

être obtenue avec un faible champ magnétique qui ne détruira pas la supraconductivité. En outre, pour rendre le système unidimensionnel, on utilise des nanofils semi-conducteurs à fort couplage spinorbite tels que l'antimoniure d'indium (InSb) ou l'arséniure d'indium (InAs). De telles structures hybrides, réalisées avec un nanofil enrobé d'un supraconducteur standard comme l'aluminium ou le niobium et dont les propriétés sont étudiées sous champ magnétique, ont été réalisées par plusieurs groupes à la suite des propositions théoriques $[6,7]$.

Pour démontrer que la supraconductivité induite est topologique, les expérimentateurs cherchent alors à détecter l'apparition de quasi-particules de Majorana à énergie nulle. L'expérience la plus simple consiste à mesurer le courant qui passe à travers un contact métallique faiblement couplé à l'extrémité du nanofil enrobé de matériau supraconducteur, sous l'effet d'une tension de polarisation entre le métal et le nanofil. Un courant peut passer uniquement si des états à l'extrémité du nanofil sont disponibles pour accueillir les quasi-particules provenant du métal. Dans les supraconducteurs standard, le gap détermine une tension-seuil en deçà de laquelle aucun courant ne circule à suffisamment basse température. En revanche, la présence de quasi-particules de Majorana associées à un état d'énergie nulle permet au courant de circuler même à faible tension. De plus, les théoriciens prédisent que la conductance correspondante atteint le quantum de conductance $2 e^{2} / h$, quel que soit le couplage entre le métal et le supraconducteur. Une telle quantification de la conductance est d'ailleurs une autre manifestation de la topologie. Un courant à faible tension a effectivement été observé dans un nanofil lorsque le champ magnétique dépasse une valeur critique déterminée par la levée de dégénérescence de spin (fig. 4) [8]. Des signatures de quasiparticules de Majorana dans la conductance électrique ont également été observées dans d'autres systèmes tels que des chaines d'atomes magnétiques de fer déposés sur du plomb qui est un supraconducteur avec un fort couplage spin-orbite [9], ou encore un supraconducteur conventionnel déposé sur un isolant topologique bidimensionnel possédant un état de bord métallique unidimensionnel [10]. Par contre, pour exclure toute explication alternative de ces effets, d'autres signatures sont requises.

On peut par exemple exploiter les effets électrostatiques. Considérons un nanofil enrobé d'un supraconducteur et faiblement contacté à des électrodes métalliques qui permettent d'appliquer une tension de polarisation entre les deux extrémités du nanofil. La charge électrique portée par ce nanofil est un multiple bien défini de la charge élémentaire; elle peut être contrôlée par une grille électrostatique, comme dans un condensateur. Cette charge est donc fixée la plupart du temps, et le courant électrique qui traverse le nanofil est bloqué. En variant la tension de grille, il est cependant possible de rendre deux états de charge différente dégénérés en énergie. Dans ce cas, un nouveau régime de transport électrique impliquant l'état formé par les deux quasi-particules de Majorana localisées à chaque extrémité du nanofil a été mis en évidence [11]. Les chercheurs ont mesuré la protection topologique associée à la façon dont cet 
état s'écarte du niveau de Fermi quand le nanofil est de plus en plus court. Cette expérience préfigure des protocoles pour mesurer si un état d'énergie nulle est vide ou occupé.

Une autre possibilité consiste à mesurer le courant à travers une jonction formée par deux supraconducteurs entre lesquels une tension de polarisation continue est appliquée. L'échange cohérent de paires de Cooper entre deux supraconducteurs conventionnels produit un courant alternatif qui oscille à une fréquence proportionnelle à la tension. Cet effet prédit par Josephson, et pour lequel il a reçu le prix Nobel de physique en 1973, a joué un grand rôle dans la validation des théories microscopiques de la supraconductivité et a de nombreuses applications en métrologie. Lorsque les supraconducteurs formant la jonction sont topologiques, la nature fractionnaire des quasi-particules de Majorana qui s'hybrident de part et d'autre de la jonction se traduit par une réduction de moitié de la fréquence Josephson. Des signatures compatibles avec un tel effet ont également été observées [12].

\section{Perspectives pour le calcul quantique topologiquement protégé}

La prochaine étape consistera à manipuler ces quasi-particules de Majorana. Leur potentiel apparait en effet lorsqu'un grand nombre d'entre elles peut être créé. Comme discuté ci-dessus, une paire de quasi-particules de Majorana correspond à un état fermionique ordinaire non local à énergie nulle. Une telle paire de quasiparticules de Majorana forme donc un système à deux états dégénérés qui peut réaliser un bit quantique - ou " qubit "; par exemple, l'état vide représente le ' $O$ ' logique, tandis que l'état occupé représente le ' 1 ' logique. Ainsi, en construisant un réseau de supraconducteurs unidimensionnels, on peut disposer de $2 \mathrm{~N}$ quasi-particules de Majorana correspondant à $N$ états fermioniques non locaux d'énergie nulle qui peuvent être vides ou occupés. À parité fixée, l'état fondamental du système a donc une dégénérescence $2^{N-1}$ qui devient extrêmement grande quand $N$ croît. Un tel système se comporterait alors comme $N-1$ bits quantiques, qui sont aujourd'hui activement étudiés pour envisager une alternative quantique à l'informatique

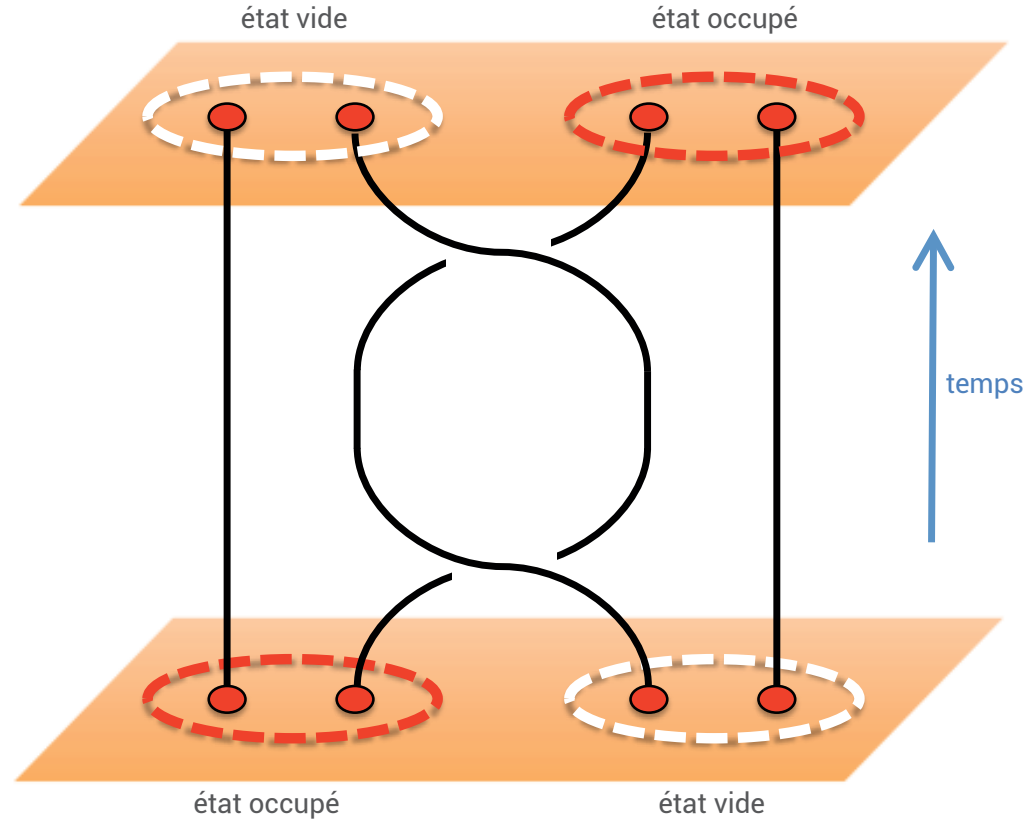

5. L'occupation d'un état formé par une paire de quasi-particules de Majorana peut être manipulée en opérant des échanges entre leurs positions. La figure montre les trajectoires de quasi-particules de Majorana qui mènent à un transfert de l'occupation d'une paire à une autre. Pendant tout le processus, les quasi-particules de Majorana doivent rester loin les unes des autres. Ce type de processus permet de concevoir des portes logiques avec des qubits constitués par des paires de quasi-particules de Majorana, sans perdre la protection topologique conférée par la non-localité.

classique omniprésente dans notre environnement. De surcroit, cette réalisation de qubits bénéficierait de la protection topologique associée à la non-localité intrinsèque des objets qui les constituent ; cela lui conférerait un avantage majeur par rapport à d'autres propositions de qubits.

La manipulation des quasi-particules de Majorana est possible car, contrairement aux particules imaginées par Majorana, ce ne sont pas des fermions. Ce ne sont pas davantage des bosons, mais ce qu'on appelle des " anyons ». Ce type de particule ne peut pas exister en tant que particule élémentaire, mais peut être réalisé comme quasi-particule dans un solide. $\mathrm{Si}$ on échange les positions de deux fermions ou de deux bosons dans un système quantique, celui-ci revient dans le même état. Cela reste vrai pour les anyons abéliens. (Les bosons, les fermions et les anyons abéliens se distinguent par la phase qu'acquiert la fonction d'onde quantique qui les décrit lors de l'échange de deux d'entre eux.) En revanche, la situation est différente pour les anyons non-abéliens, dont font partie les quasi-particules de Majorana, pour lesquels un échange peut mener à un état quantique différent. En opérant des échanges entre les positions de $2 \mathrm{~N}$ quasiparticules de Majorana, il est alors possible d'effectuer des "opérations " quantiques dans le sous-espace dégénéré des $2^{N-1}$ états propres qu'ils déterminent [13]. Ce jeu de bonneteau quantique revient à faire apparaitre et disparaitre des billes cachées sous des gobelets déplacés sur une table, mais sans tricher en soulevant ces gobelets! Une telle expérience serait la première démonstration que les quasi-particules de Majorana sont utiles pour réaliser certaines opérations de l'information quantique (fig. 5).

Pour effectuer ces échanges, différentes géométries ont été proposées. Les expérimentateurs commencent à synthétiser des réseaux formés de plusieurs nanofils enrobés d'aluminium supraconducteur (voir par exemple l'image du haut de la page 5), en espérant que des quasi-particules de Majorana apparaissent à chaque extrémité 
de ces nanofils. Leur manipulation serait obtenue grâce à l'application de grilles électrostatiques et de flux magnétiques. En particulier, les effets électrostatiques discutés ci-dessus permettent de concevoir un qubit formé de quatre quasi-particules de Majorana, dont le '0' logique et le '1' logique correspondent à des états de même parité. Grâce à la répulsion coulombienne, un tel qubit topologique serait protégé des charges électriques mobiles dans son environnement. Il serait donc potentiellement plus stable.

Le domaine de recherche qui vient d'être décrit s'est développé dans un temps relativement court. Les premiers travaux théoriques datent des années 2000 ; ils ont été rapidement suivis de propositions concrètes d'architectures à réaliser en laboratoire. Les premiers résultats expérimentaux sont ensuite apparus en 2012. Une telle rapidité ne doit cependant pas occulter que ce domaine de recherche a profité de la maturité des micro- et nanotechnologies développées depuis les années 1980 , et notamment leur application au domaine de la supraconductivité mésoscopique et des effets de proximité. Les fortes contraintes sur les paramètres à réaliser pour atteindre le régime topologique ont également nécessité une recherche poussée sur les matériaux, couteuse en moyens et en main d'œuvre. De très nombreux laboratoires publics se sont engagés dans ce domaine. Les perspectives en information quantique, qui expliquent d'ailleurs pour une large part l'engouement actuel pour la mise en évidence et la manipulation des quasi-particules de Majorana dans les supraconducteurs, ont également motivé le retour de grandes entreprises privées dans le financement d'une telle recherche fondamentale, encore très éloignée des applications. C'est le cas par exemple de Microsoft, qui a investi des moyens importants dans ce domaine en créant des laboratoires nommés «Station $\mathrm{Q}$ » à Delft, Copenhague et Santa Barbara, tandis que Google et IBM ont fourni un effort comparable sur la voie alternative des circuits quantiques supraconducteurs.

Alors que plusieurs résultats spectaculaires décrits ci-dessus ont déjà été obtenus, la qualité exceptionnelle des échantillons réalisés a aussi permis d'aborder des questions en dehors du champ de la supraconductivité topologique et que la recherche en physique mésoscopique avait laissées en

\section{Références}

1• E. Majorana, "Teoria simmetrica dell'elettrone e del positrone", Nuovo Cimento 14 (1937) 171.

2. C. Marquet et L. Simard, « Le neutrino est-il identique à son antiparticule ?», Reflets de la physique, 24 (2011) 11.

3. N. Read et D. Green, "Paired states of fermions in two dimensions with breaking of parity and time-reversal symmetries, and the fractional quantum Hall effect", Phys. Rev. B 61 (2000) 10267.

4• A.Yu. Kitaev, "Unpaired Majorana fermions in quantum wire", Phys. Usp. 44 (2001) 131.

5 L. Fu et C.L. Kane, "Superconducting proximity effect and Majorana fermions at the surface of a topological insulator", Phys. Rev. Lett. 100 (2008) 096407.

6• R.M. Lutchyn et al., "Majorana Fermions and a topological phase transition in semiconductor-superconductor heterostructures", Phys. Rev. Lett. 105 (2010) 077001.

$7 \cdot$ Y. Oreg et al., "Helical liquids and Majorana bound states in quantum wires", Phys. Rev. Lett. 105 (2010) 177002.

8. V. Mourik et al., "Signatures of Majorana fermions in hybrid superconductor-semiconductor nanowire devices", Science 336 (2012) 1003.

9. S. Nadj-Perge et al., "Observation of Majorana fermions in ferromagnetic atomic chains on a superconductor", Science 346 (2014) 602.

10 Q.L. He et al., "Chiral Majorana fermion modes in a quantum anomalous Hall insulator-superconductor structure", Science 357 (2017) 294.

11 • S.M. Albrecht et al., "Exponential protection of zero modes in Majorana islands", Nature 531 (2016) 206

12• L.P. Rokhinson et al., "Observation of the fractional ac Josephson effect: the signature of Majorana particles", Nature Phys. 8 (2012) 795.

13. D.A. Ivanov, "Non-abelian statistics of half-quantum vortices in p-wave superconductors", Phys. Rev. Lett. 86 (2001) 268.

14• H. Zhang et al., "Quantized Majorana conductance", Nature 556 (2018) 74.

suspens, faute de matériaux adaptés. D’un point de vue conceptuel, ce domaine a naturellement accompagné un très large mouvement d'ensemble pour la prise en compte des aspects topologiques dans de multiples domaines de la physique. La topologie a d'ailleurs été mise en avant avec le prix Nobel de physique attribué à Haldane, Kosterlitz et Thouless en 2016. On peut donc raisonnablement espérer que des découvertes majeures continueront d'apparaitre suite aux efforts dans ce domaine, en lien avec les prédictions de Majorana... ou pas. (a) Cet article est la version largement développée d'une contribution que nous avions rédigée pour le dossier "Les formes exotiques de la matière " du n530 de La Recherche (décembre 2017).

(b) La conductance est l'inverse de la résistance.

(c) Le caractère pair ou impair est déterminé par les états loin du niveau de Fermi qui ne participent pas au condensat. 